BNL 36278

BNL- -36278

DE85 010954

THE AGS BROAD BAND NEUTRINO BEAM

D.H. White - Physics Department

February 27, 1985

\title{
DISCLAIMER
}

as an account of work sponsored by an agency of the United States This report was prepared as an account of wovernment nor any agency thereof, nor any of their Government. Neither the Untted States Government nor assumes any legal liability or responsiemployees, makes any warranty, express or impleed, or ans information, apparatus, product, or bility for the accuracy, completeness, or usefulness of any ininge privately owned rights. Referprocess disclosed, or represents that its use would nol ins, or service by trade name, trademark, ence herein to any specific commercial produce, procass or sericely its endorsement, recommanuficturer, or otherwise does not necessarily constiment or any agency thereof. The views mendation, or favoring by the United States Goverame necessarily state or reflect those of the

and opinions of authors expro ssed herein do not
United States Government or any agency thereof. 


\section{THE AGS BROAD BAND NEUTRINO BEAM}

\section{Introduction}

We describe the broad band neutrino beam in the north area of the AGS and discuss the calculation of the neutrino flux. The horns were initially designed by Robert Palmer and this beam has been used for almost all neutrino running at the AGS. All of the wide band running for E734 has been done in the beam we discuss below. E734 is an experiment designed to measure elastic scattering of neutrinos and antineutrinos on electrons and protons. The detector is sufficiently large ( $s 170$ tons) that enough events can be detected to make precision measurements of cross sections. In particular, the reaction

$$
v_{\mu}+e+v_{\mu}+e
$$

has been detected with more than 100 events ${ }^{l}$, requiring a detailed understanding of the beam characteristics for normalizarion. The normalization method uses the quasi elastic reactions

$$
\begin{aligned}
& v_{\mu}+n+\mu^{-}+p \\
& \bar{v}_{\mu}+p+\mu^{+}+n
\end{aligned}
$$

These cross sections are relatively well known, below $1 \mathrm{GeV}$ they rise approximately linearly with neutrino energy and then become rather independent of neutrino energy above $1 \mathrm{GeV}$. The approach to constant behavior is quite different between $\nu$ and $\bar{v}$ so that accurate relative normalization requires a detailed 
knowledge of the $v_{\mu}$ spectrum of both polarities. The study of neutrino oscillations between $\nu_{\mu}+\nu_{e}^{2}$ requires a systematic understanding of the inherent $v_{e}$ flux in the $\nu_{\mu}$ beam and this problem we address also in this report.

The calculation of absolute $\nu$ flux and the comparison with measured data is even more difficult than the relative comparisons we allude to $a^{3}$ e. The method that is used rests on our understanding of the - rged current quasi elastic reactions. ${ }^{3}$ The charged current cross section is related to the electron scattering data through CVC and the major uncertainty is in the value of the axial vector mass $m_{A}$. Knowledge of this mass which is obtained from angular distribution measurements, allows an absolute calculation of the cross section. The magnitude of the cross section together with the event distribution permits an absolute determination of the $v$ flux. Although the physics of the situation is clear, technical difficulties have limited the agreement between calculation and measurement to the $20 \%$ level. As we expect to show, the understanding of this neutrino beam has progressed enough that relative normalizations appreciably better than this are possible.

Beam Hardware

A schematic of the target area is shown in Fig. 1. The proton beam is extracted in a single turn from the AGS and transported down to the target of Fig. 1. The incident proton beam energy has been $28.3 \mathrm{GeV} / \mathrm{c}$ and the spot size at the target has been typically $2 \mathrm{~mm}$ full width. The tine structure of the beam fron the accelerator RF 
is preserved, and so 12 bunches 224 ns apart appear at the target. The width of each bunch is about $30 \mathrm{~ns}$. This time structure is invaluable in separating events induced by neutrinos and events which originate from less direct sources. The overall spill is just a little longer than $2.5 \mu$ sec.

The secondary particles that originate in the target are focussed in the B field of the two horns as we describe in the next section. The $B$ field is generated by a current of $=200-300 \mathrm{KA}$ which flows in the horn conductor. A simple schematic of the power supply is shown in Fig. 2. The energy storage capacitors, C, are charged in the $1.4 \mathrm{sec}$ between pulses to $=12 \mathrm{kV}$ at constant current. The capacitors are arranged in modules $42 \mu \mathrm{F}$ each and each module is switched on to the load through an ignitron - che resistor $R_{L}\left(5 \times 10^{-3} \Omega\right)$ is in series with 14 transmission 1 ines in parallel driving the horns $\mathrm{H} l$ and $\mathrm{H} 2$ in series through a characteristic impedance of about $1 \Omega$. Inmediately after the horn current is switched on, the system acts as a series RLC circuit with a time constant of $\approx 20 \mathrm{usec}$. The ignitrons are triggered by a pulse derived from the AGS radio frequency, which implies that the horn current pulse is stable with respect to beam time to about $20 \mathrm{~ns}$. The current pulse is shown in Fig. 3. When the current falls ro zero the ignitrons 12 crowbar the system preventing reverse voltage from being developed across II. The current is monitored precisely in one module and approximately in the other eleven. The charging time represents a monitor of the functioning of all twelve modules, in that it is linearly dependent on the number of modules that are recharged. 
In the shield at the end of the decay region, ion chambers are installed which monitor the muon flux in the shield. These chambers are primarily used to monitor the beam alignment although the radial distribution of the muons agrees with the results of the NUBEAM calculation.

\section{Horn Optics}

A schematic diagram of the wide band horns is shown in Fig. 4. The target is embedded in the front of horn 1 and the charged particles that are produced in the target are reduced in angular spread between the two horns. The second horn then produces a nearly parallel beam so that the length of the particle trajectory in the decay region is maximized. In the simplest terms, the horn functions as a simple lens and for this to be true the length of the horn as a function of radius is important. The B field at a distance from a current carrying conductor varies as $1 / r$; for focussing to occur, the angular change of the charged particle must vary as $r$ so that $\int B d l$ must also vary as $r$. The horn length must vary approximately as $r^{2}$ therefore. This geometry can only produce a parallel output beam at one energy, and since we are usually concerned with maximizing the charged particle flux over an energy band, the variation of aperture with charged particle momentum is important.

The production cross section $d \sigma / d \Omega=\exp -a p_{1}^{2}$, a function which falls rapidly with angle. The number of particles which are produced in an annulus $d \theta$ at $\theta$ is $d \sigma / d \Omega \sin \theta d \theta$ which is 0 at $\theta=0$ 
and $r$ ises to a maximum at $\theta_{\max }$ for a given momentum. The horn system we are describing is designed so that particles at this production angle $\theta_{\max }$ are focussed as we have described to a parallel beam after horn 2. The chromatic properties of this lens system are optimized by this means to produce a wide band of transmitted momenta after horn 2 with the angular acceptance peaking near the optimal production angle. The horns are produced as a stepwise linear approximation to the necessary shape.

Using the NUBEAM program ${ }^{4}$ which we discuss below we have calculated the enhancement in the neutrino flux produced by this horn system over the bare target spectrun without focussing. The central momentum of this horn system at $250 \mathrm{RA}$ current is expected to be at $3 \mathrm{GeV} / \mathrm{c}$. For $\pi \rightarrow \mu+v_{\mu}$, the neutrino momentum is $\$ .42 \mathrm{p}_{\pi}$ in the forward direction so that we would expect the maximal enhancement from the horns to be at $1.3 \mathrm{GeV} / \mathrm{c}$. In Fig. 5 we show the calculated enhancement with a broad peak centered at $1.3 \mathrm{GeV} / \mathrm{c}$ and extending to high momenta. At low momenta, the particles are over focussed and are deflected so as to strike the walls of the enclosure and be absorbed before they can deray. Also in Fig. 5 is the effect of the horr for the "wrong sign" particles. Near the optinal point we notice that the effect of the horns is actually to reduce the number of charged particles that are transmitted as we would expect. At high momenta there is also a moderate enhancement which will ultimately decrease to one at the highest momenta, when the bending effect of the horns becomes negiigible. 
The enhancement drops rapidly at low momenta partly from momentum dependent effects and also because the optimal production angle moves outside the aperture of the system. This production angle is given by $\mathrm{P}_{\perp}=0.25 \mathrm{GeV} / \mathrm{c}$ so that below $1.5 \mathrm{GeV} / \mathrm{c}$ pion womenta the fraction of particles that are transmitted rapidly decreases, this combined with the chromatic effects produces a very rapid fall off at low momenta. Moreover the rapidity of the fall off causes this region of the spectrum to be very dependent on the details of the calculation.

\section{Particle Sources}

Both charged $\pi$ and $k$ mesons produce neutrinos through their decay. The ratio of the production rate of $\pi$ and $k$ differs by about a factor of 20 but as we shall see below the neutrinos from $K$ decay appear at higher momentum and are observable at the high end of the spectrum. The predominant branching mode of the $\pi$ meson is $\pi \rightarrow \mu+$ $\nu_{\mu}$ and at the relevant energies any reasonable detector only detects neutrinos close to the forward direction. The kinematics is such that

$$
E_{\nu}=\frac{m_{\pi}^{2}-m_{\mu}^{2}}{m_{\pi}^{2}+p_{\pi}^{2} \theta_{\nu}^{2}} \quad \cdot p_{\pi}
$$

so that when $\theta_{V}=0 E_{V}=0.42 \mathrm{P} \pi$ and at the maximum angle for neutinos in the $\mathrm{E} 734$ detector (33mr) the neutrino momentum drops to about . $28 \mathrm{P} \pi$, about two thircis of the maximum. The neutrino spectrum from $\pi-\mu$ decay is a slightly broadened version of the 
pion spectrum that is transmitted by the horn system modified by the decay probability. The central. momentum of the pions is $3 \mathrm{GeV} / \mathrm{c}$ and so that $\gamma c \tau$ is about $160 \mathrm{~m}$, much longer than the decay region of the horn, and so a small fraction of the pions decay before reaching the shield. The $v_{\mu}$ spectrum from pion decay is shown in Fig. 6. The 1 ifetime of the muon is $2200 \mathrm{~ns}$ and on the average the muons traverse only one half of the decay distance. The ratio of the fraction of muons that decay to the pion decays is $2 \times 10^{-3}$. This gives rise to a contamination of electron neutrinos which is shown in Fig. 7. The $v_{e}$ spectrum is similar to the $v_{\mu}$ spectrum but lower in energy by about a factor of two.

The $K$ mesons contribute to the $v_{\mu}$ spectrum primarily tingugh the $\mathrm{K}_{\mu 2}$ decay mode. The kinematics are similar to the two body pion decay except that the forward neutrinos are very close to the charged kaon momentum throughout the angular range of the E734 detector. The $v_{\mu}$ spectrum for $\mathrm{K}^{+}$decay is shown in Fig. 6 together with the tota: of $\pi$ and $k$. The spectrum from $k_{\mu_{2}}$ is significantly harder as one would expect from the kinematics. The low energy enhancement in the $\mathrm{K}^{+}$induced spectrum is due to the secondary decay of $\pi$ mesons from the decay mode $\mathrm{K}^{+} \rightarrow \pi^{+}+\pi^{\circ}$.

The branching mode $\mathrm{K}^{+}+\pi^{\circ}+\mathrm{e}^{+}+\nu_{e}$ gives $\nu_{e}$ directly with a branching ratio of .05 . The energies of $v_{e}$ from this mode are spread over the entire spectrum up to the end point with a typical neutrino energy of about one third of the $\mathrm{K}^{+}$energy. The production rate of $\mathrm{K}^{+}$is about .05 of $\pi^{+}$, the branching ratio is .05 but the 
ratio of $Y c t$ for kaon and pion is 7.5 . These combine to give an expected $v_{e} / v_{\mu}$ ratio of $6 \times 10^{-3}$ for the entire beam spectrum. We show in Fig. 7 the $v_{e}$ spectrum from $k^{+}$decay with the characteristics we describe.

The contribution of other particles to the $v_{\mu}$ spectrum is rather small and is customarily neglected. However in the case of $v_{e}$ since the major sources are already small we need to consider other possibilities in arriving at a careful estimate of the $v_{e}$ component. The principal contribution other than those we have described comes from $K_{L}{ }^{\circ}$ decay. Although $K_{L}{ }^{\circ}$ do not have the enhancement of the charged particle focussing, Yct is comparable with the pion value and the branching ratio to $k \rightarrow \pi e v$ is 0.40 . We show the yield of $v_{e}$ from $K_{L}^{\circ}$ in Fig. 7 , an identical contribu tion of $\bar{v}_{e}$ is also present. The total $v_{e}$ spectrum is also in Fig. 7 .

NUBEAM

The yields in Figs. 6,7 were calculated using a program NUBEAM from CERN. ${ }^{4}$ We discuss the program here in general terms with an emphasis on the possible cncertainties. The beam elements, target, horns, shield, etc. are specified by piecewise linear models to any degree of detail that one has the patience to introduce. The currents are specified in the horns and the $B$ field is calculated at initialization. Particles are produced in the target according to a production spectrum which is specified as a table in secondary particle momentum and angle. The particle is traced through the 
beam elements and a probability calculated for decay. A decay stack is set up specifying the possible modes of decay of all the particles. The weighted probabilities are stored for subsequent plotting. The muons are traced through the shield and multiple scattering is included in the ray tracing.

Hadrons are produced with a distribution specified by the included spectra ${ }^{6}$ and then subsequently absorbed according to cross sections appropriate to the material being traversed. The absorption cross section as usually defined ${ }^{5}$ includes protons which scatter through angles small enough to remain in the target and which may then interact. Since the majority of protons that interact will have the potential of reinteracting either in the target or the inner conductor of the horn, the cross sections of Ref. 5 are appropriate.

The protons which remain after interaction do so with reduced energy. The loss of proton energy has a signifjcant effect on the momenta of the secondary pions. To calculate this process exactly would entail creating a model of the hadron cascade which is prohibitive for the beam calculation. An alternative procedure which we have followed is to make an estimate of the production of secondary pions from reinteracting protons and add it to the production spectrum of the pions from the original beam. The composite spectrum is also reproduced in Table $Z$ for both $\pi^{+}$and $\pi^{-}$production. This yield is compatible with that from charged particle beams at the AGs. ${ }^{7}$ 
These considerations are not important for the secondary particles that traverse material in the horns and beam line because that material is a small fraction $(* 10 \%)$ of an interaction length and the effect is negligible.

Comparison with Measured Data

The neutrino beam spectrum has been measured with three techniques described in detail in Ref. ${ }^{8}$. We show in Fig. 8 a composite neutrino spectrum together with the results of the calculation. The agreement is seen to be good although the freedom that was used in estimating the effective secondary flux should be noted. The effect of the protons that are degraded in energy producing secondary particles is important in achieving the agreement of calculation and measurement that is shown here.

Acknowledgments

This work was part of experiment E734 at the AGS. My colleagues are 1 isted in reference 1 and a more comprehensive account of the analysis of the spectra is being prepared as reference 8 . It is a pleasure to acknowledge the collaboration with Roger Carlini in understanding NUBEAM.

This research has been supported by the U.S. Department of Energy under Contract DE-AC02-76CH00016. 


\section{$\underline{\text { References }}$}

1. L.A. Ahrens et al. Phys. Rev. Lett. 51, 1514 (1983).

2. L.A. Ahrens et al. Phys. Rev. to be published.

3. For example, N.J. Baker et al. Phys. Rev. D25, 2495 (1981). For a discussion of the determination of the quasi elastic cross section and for a neutrino spectrum.

4. C. Visser, NUBEAM Neutrino Bear Simulator, Hydra Applications Library. CERN 1979, modified extensively by R. Carlini, Los Alamos National Laboratory.

5. A.s. Carro11 et a1. Phys. Lett. 80B, 319 (1979).

6. The spectra that were used as an initial data for the calculation were from Particle Spectra, H . Grote, R. Hagedorn \& J. Ranft, CERN 1970. The spectra of R. Sanford \& C.L. Wang, BNL 11479, 1967 (unpublished) were also used for comparison.

7. BNL 50874, G. Bunce, May 1978, AGS Beams.

8. L.A. Ahrens, et al. (BNL - OG 781). Pecise Determination of the Neutrino Fluxes in the Brookhaven Broad Band Beam, to be subm. to Phys. Rev. D. 


\section{Figure Captions}

Fig. 1. A schematic of the neutrino beam area. The slots for muon detectors are shown in the shield. In the neutrino detector enclosure, tire main detector, the gamma catcher and muon spectrometer are shown.

Fig. 2. The capacitors $C$ are $42 \mu F$, the inductance of the two horns in series is $1.25 \mu \mathrm{A}$ and the load resistor $\mathrm{R}_{\mathrm{L}}$ is 5 $\times 10^{-3} \Omega$.

Fig. 3. The current pulse on the horn system at $20 \mu \mathrm{sec} / \mathrm{cm}$.

Fig. 4. A schematic of che conductor geonetry of the horn system. The target is embedded in the throat of horn 1 . Note that the axial and radial scales differ.

Fig. 5. The enhancement factor produced by the horn system at $250 k A$ as a function of neutrino momentur. at the detector.

Fig. 6. The $v_{\mu}$ spectrum at the detector as a function of the neutrino energy. The spectrum is integrated over a radius of $1.5 \mathrm{~m}$ and the particle production spectrum is that of Table I.

Fig. 7. The $\nu_{e}$ spectrum as a function of neutrino energy integrated over a radius of $1.5 \mathrm{~m}$.

Fig. 8. The $\nu_{\mu}$ spectrum as a function of neutrino energy both calculated and measured.

Fig. 9. The $v_{e}$ spectrum as a function of neutrino energies, both calculated and measured. 
000000000000000000000000000000000000000000000000000000

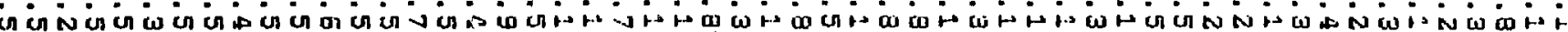

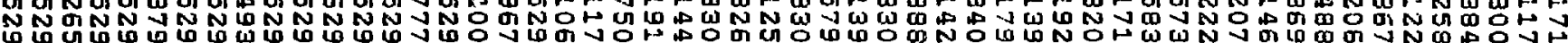

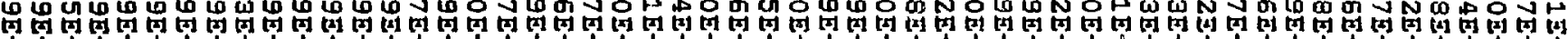

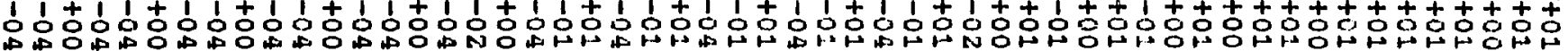
000000000000000000000000000000000000000000000000000000

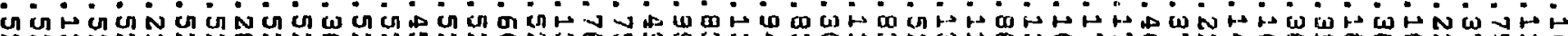

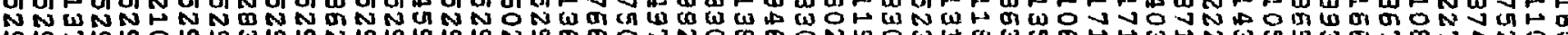


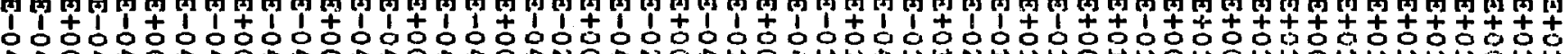

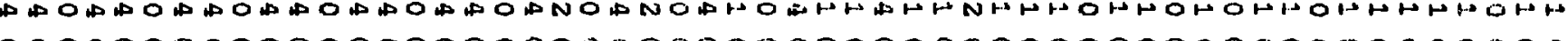
000000000000000000000000000000000000000000000000000000 ज G

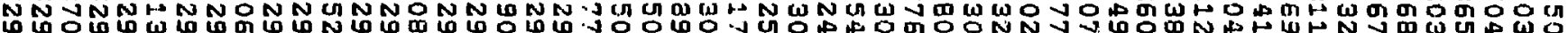

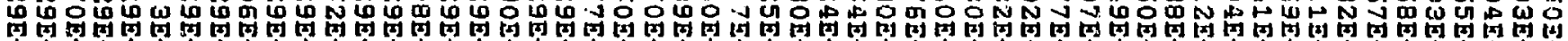
1
0
0 000000000000000000000000000000000000000000000000000000 क of

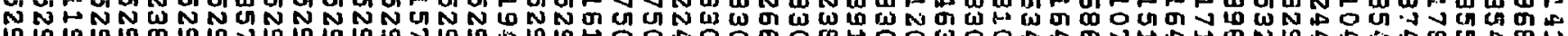

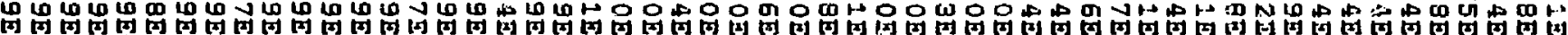

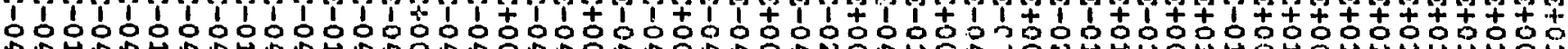
0000000000000

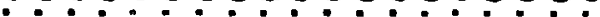

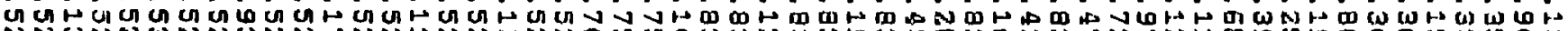
NNWNNWNNWNN NNN NNN N

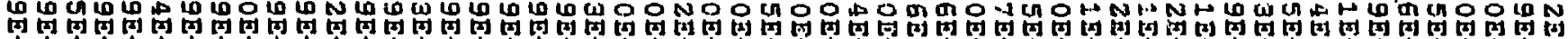

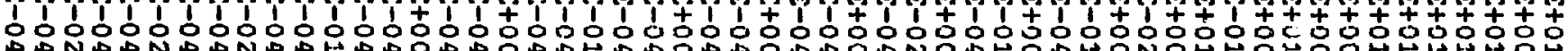
000000000000000000000000000000500000000000000000000000

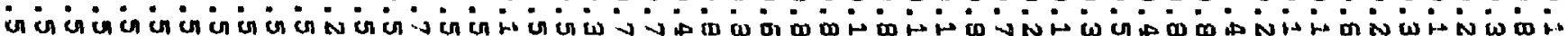

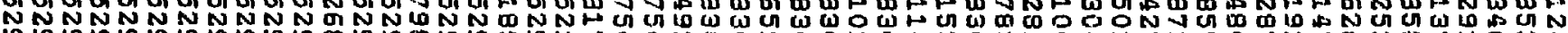

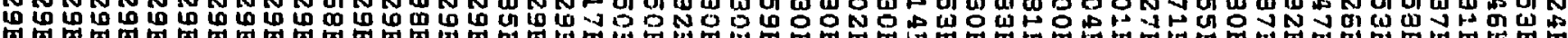

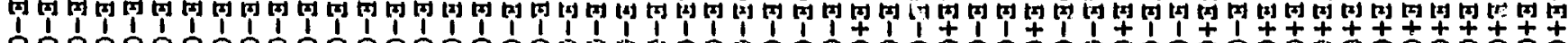

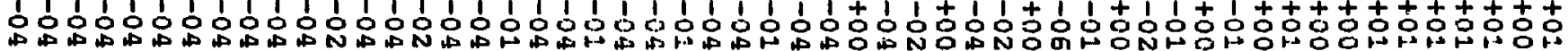


Negative pion Spectra

Angles start at 0.5 degree increment by 1 degree 18 steps Momenta start at $0.5 \mathrm{Gev}$, increment by $1 \mathrm{Gev} 13$ steps
$0.3765+00$
$0.365 \Sigma+00$
$0.341 E+00$
$0.314 \mathrm{E}+00$
$0.2882+00$
$0.2545+00$
$0.243 E+00$
$0.22 E E+00$
$0.21 a E+00$
$0.199 E+00$
$0.17 \mathrm{BE}+00$
$0.256 E+00$
$0.155 \mathrm{E}+00$
$0.142 E+00$
$0.131 \Sigma+00$
$0.122 \Xi+00$
$0.113 \mathrm{D}+00$
$0.103 E+00$
$0.250 \mathrm{E}+01$
$0.7 B 4 E+00$
$0.138 \mathrm{E}+\mathrm{c}$
$0.127 \mathrm{E}+01$
$0.114 \bar{I}+01$
$0.100 E+01$
$0.3845+00$
$0.332 E+00$
$0.294 \mathrm{E}+00$
$0.58 B E+00$
$0.499 E+00$
$0.438 \Sigma+00$
$0.285 E+01$
$0.277 \mathrm{E}+01$
$0.259 E+01$
$0.255 E+00$
$0.240 E+00$
$0.215 E+00$
$0.132 E+03$
$0.103 E+01$
$0.798 E+00$
$0.231 E+01$
$0.198 E+01$
$0.1632+01$
$0.277 E+00$
$0.207 E+00$
3.156E+00
$0.46 a E+00$
$0.357 \mathrm{E}+00$
$0.227 \mathrm{E}+01$
$0.217 \mathrm{E}+01$
$0.205 E+01$
$0.1235+00$
$0.102 E+00$
$0.798 E-01$
$0.100 E+01$
$0.598 \mathrm{E}+00$
$0.479 E+00$
$0.167 \mathrm{E}+01$
$0.177 \mathrm{E}+01$
$0.135 \mathrm{E}+01$
$0.1 \pm 0 E+00$
$0.743 \mathrm{a}-01$
$0.523 E-01$
0. 357 5-0:
$0.223 E+00$
$0.152 E+00$
$0.158 E+01$
$0.113 \mathrm{E}+01$
$0.996 \bar{E}+00$
$0.108 \mathrm{a}+01$
$0.22 \mathrm{CE}-03$
$0.53 i 5-02$
$0.525 E+00$
$0.342 E+00$
$0.207 E+00$
$0.127 \mathrm{E}+00$
$0.110 \Xi+01$
$0.779 \mathrm{E}+00$
$0.291 E-01$
$0.166 \mathrm{E}-01$
$0.105 E-01$
$0.627 E-02$
$0.7665-01$
$0.456 \mathrm{E}-01$
$0.205 E+01$
$0.142 E+01$
$0.116 E+01$
$0.117 E \div 01$
$0.213 \mathrm{E}-02$
$0.830 \mathrm{E}-04$
C. 299E+00
$0.170 \mathrm{E}+00$
$0.930 E-01$
$0.798 \mathrm{E}+00$
$0.509 E+00$
$0.627 \mathrm{E}-02$
$0.213 E-02$
$0.830 E-04$
$0.519 E-01$
$0.249 \mathrm{E}-\mathrm{C1}$
$0.105 E-01$
$0.188 E+01$
$0.173 E+01$
$0.830 E-04$
$0.830 E-04$
$0.134 \mathrm{E}+01$
$0.88 B E+00$
$0.554 E+00$
C. 830E-04
$0.156 \mathrm{E}+00 \quad 0.763 \mathrm{E}-0 \mathrm{i}$
$0.373 E-01$
$0.1665-01$
$0.421 E-02$
$0.305 \Sigma+00$
$0.830 \mathrm{E}-04$
$0.8305-04$
$0.83 \mathrm{CE}-04$
$0.330 \mathrm{E}-04$
$0.930 E-04$
$0.53 C E-04$
$0.173 E+01$
$0.110 \mathrm{E}+01$
$0 . E B 1 E+00$
$0.384 E+00$
$0.330 E-04$
$0.87: \Sigma-01$
$0.394 \mathrm{E}-0 \mathrm{i}$
0. $287 \mathrm{E}-01$
$0.627 \mathrm{E}-02$
$0.830 \mathrm{E}-04$
$0.189 E+00$
$0.83 \mathrm{CE}-0=0.830 \mathrm{E}-04$
$0.530 E-04$
$0.830 \mathrm{E}-04$
$0 . \mathrm{B} 30 \mathrm{E}-04$
$0.330 \mathrm{a}-04$
$0.252 E+01 \quad 0.126 E+01$
$0 . B 3 B E+00$
$0.477 \mathrm{E}+00$
$0.234 E+00$
$0.3305-04$
$0.435 \mathrm{E}-01 \quad 0.187 \mathrm{E}-01$
$0.8305-02$
$0.213 E-02$
$0.830 E-04$
$0.104 \Sigma+00$
$0.830 \mathrm{E}-04$
$0.330 \mathrm{E}-04$
$0.830 \mathrm{E}-04$
$0.8305-04$
$0.830 \mathrm{E}-04$
$0.830 \mathrm{E}-04$
$0.132 E+01$
$0.105 \mathrm{E}+02$
$0.647 \mathrm{E}+00$
$0.334 E+00$
$0.143 E+00$
$0.830 E-04$
0.22 日E-01
$0.330 \mathrm{~s}-02$
$0.213 E-02$
$0.830 \Sigma-04$
$0.830 E-04$
$0.5 B 15-01$
$0.330 \mathrm{E}-04$
$0.930 \mathrm{E}-04$
$0.930 \mathrm{E}-04$
$0.830 \mathrm{E}-04$
$0.830 \mathrm{E}-04$
$0.830 \mathrm{E}-04$
$0.104 E+01$
$0.780 \Sigma+00$
$0.448 E+00$
$0.214 E+00$
$0.825 E-01$
$0.830 .5-04$
$0.112 \mathrm{E}-01$
$0.750 \mathrm{E}-04$
$0.750 E-04$
$0.750 E-04$
$0.750 E-04$
$0.3195-01$
$0.750 E-04$
$0.750 \mathrm{E}-04$
$0.750 \mathrm{E}-04$
$0.750 \mathrm{E}-04$
$0.750 \mathrm{E}-04$
$0.603 E+00$
$0.425 E+00$
$0.225 E+00$
$0.952 E-01$
$0.3255-01$
$0.750 \Xi-04$
$0.393 \mathrm{I}-02$
$0.520 \mathrm{E}-04$
$0.520 \mathrm{E}-04$
$0.520 E-04$
$0.520 \mathrm{E}-04$
$0.117 \pm-01$
$0.520 \mathrm{E}-04$
$0.520 E-04$
$0.520 \mathrm{E}-04$
$0.520 \mathrm{E}-04$
$0.520 \mathrm{E}-04$
$0.520 \mathrm{z}-04$
$0.484 \mathrm{E}+00$
$0.327 \mathrm{E}+00$
$0.160 \mathrm{E}+00$
$0.582 E-01$
$0.192 \mathrm{E}-01$
$0.520 \mathrm{E}-04$
$0.134 \mathrm{E}-02$
$0.520 \mathrm{E}-04$
$0.520 E-04$
$0.520 E-04$
$0.520 \mathrm{E}-04$
$0.520 E-02$
$0.520 \mathrm{E}-04$
$0.520 E-04$
$0.520 E-04$
$0.520 E-04$
$0.5205-04$
$0.520 E-04$
$0.387 E+00$
$0.351 \mathrm{E}-01$
$0.910 \mathrm{E}-02$
$0.5205-04$
$0.520 \mathrm{E}-04$
$0.520 \mathrm{E}-04$
$0.520 E-04$
0.520 E-04
$0.520 E-04$
$0.134 E-02$
$0.520 \mathrm{E}-04$
$0.520 \mathrm{E}-04$
$0.520 \mathrm{E}-04$
$0.52 C E-04$
$0.520 \mathrm{E}-04$
$0.520 E-04$
$0.302 E+00$
$0.186 \mathrm{E}+00$
$0.770 \mathrm{E}-01$
$0.208 E-01$
$0.3935-02$
$0.520 \mathrm{E}-04$
$0.520 E-04$
$0.520 \mathrm{E}-04$
$0.520 \mathrm{E}-04$
$0.520 \mathrm{E}-04$
$0.520 \mathrm{E}-04$
0.520 z-04
$0.234 E+00$
$0.520 E-04$
$0.520 E-04$
$0.520 \mathrm{E}-04$
$0.520 \mathrm{E}-04$
$0.520 E-04$
$0.520 \mathrm{E}-04$
$0.520 E-04$
$0.520 E-04$
$0.117 \mathrm{E}-01$
$0.134 \mathrm{E}-02$
$0.520 E-04$
$0.520 \mathrm{E}-04$
$0.520 \mathrm{E}-04$
$0.520 \mathrm{E}-04$
$0.520 \mathrm{E}-04$
$0.520 \mathrm{E}-04$
$0.520 \mathrm{E}-04$
$0.185 \mathrm{E}+00$
$0.101 E+00$
$0.312 E-01$
$0.520 E-04$
$0.520 E-04$
$0.5202-04$
$0.520 E-04$
$0.520 \mathrm{E}-04$
$0.655 \mathrm{E}-02$
$0.520 \mathrm{E}-04$
$0.520 \mathrm{E}-04$
$0.5205-04$
$0.520 E-04$
$0.520 \mathrm{E}-04$
$0.520 E-04$
$0.520 E-04$
0.520 E-04
$0.520 \mathrm{E}-04$
$0.520 E-04$
$0.520 E-04$
$0.520 \Sigma-04$
0.520 E-04
$0.520 E-04$
$0.520 E-04$
$0.520 \mathrm{E}-04$
$0.520 \mathrm{E}-04$ 


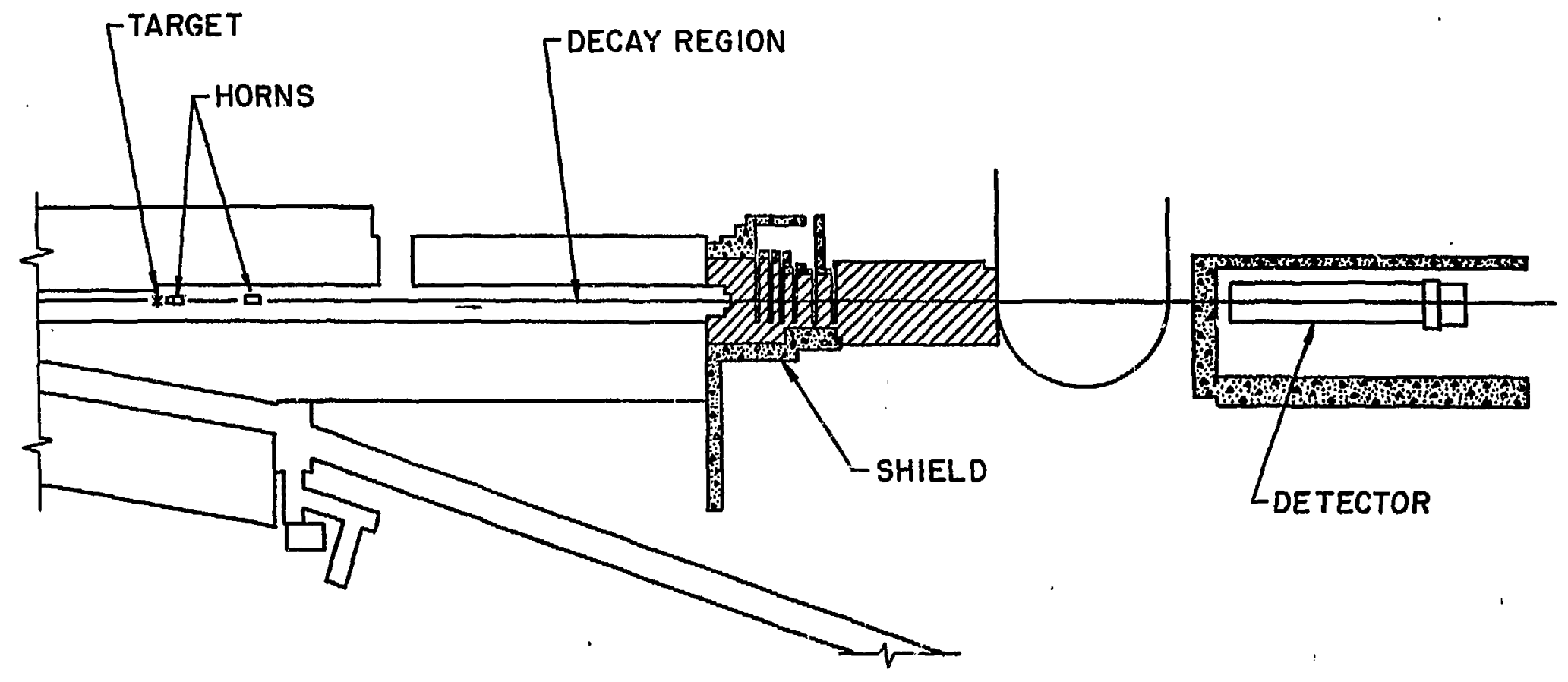




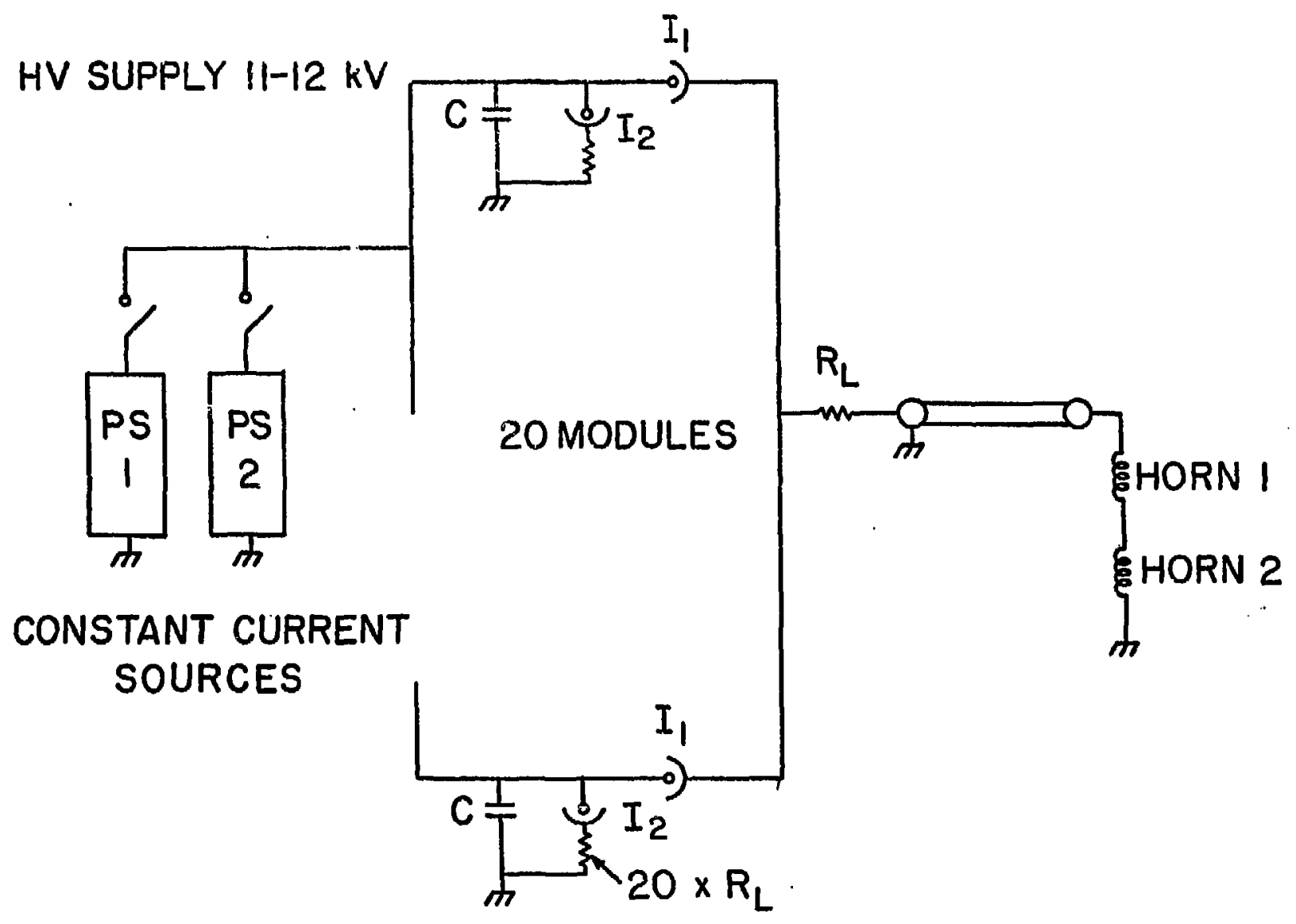




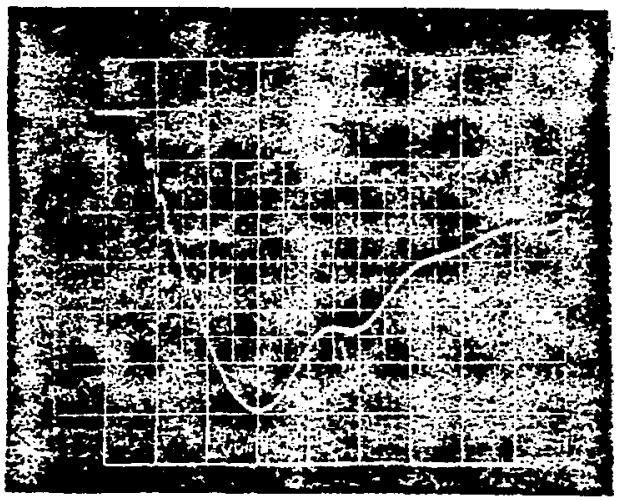




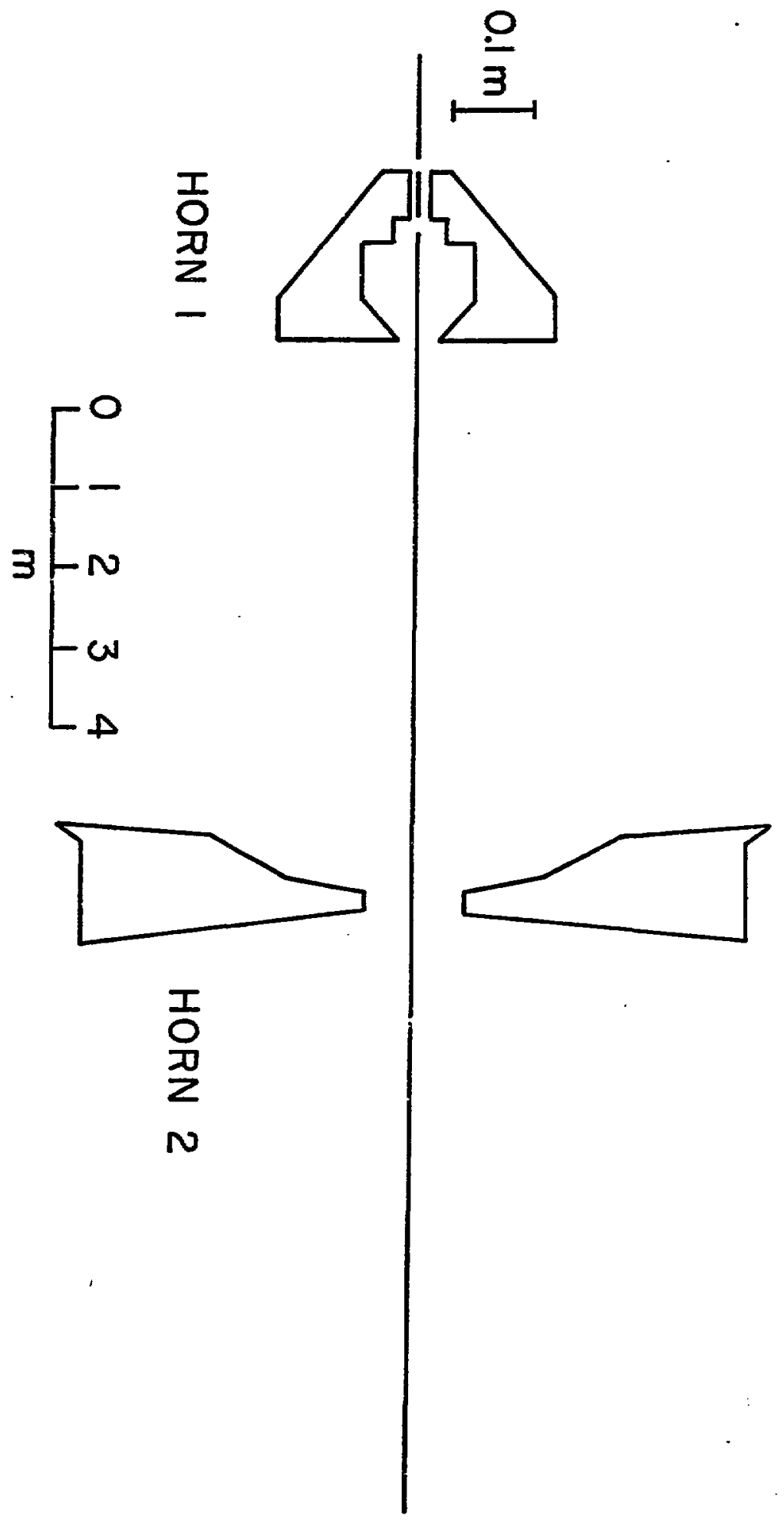




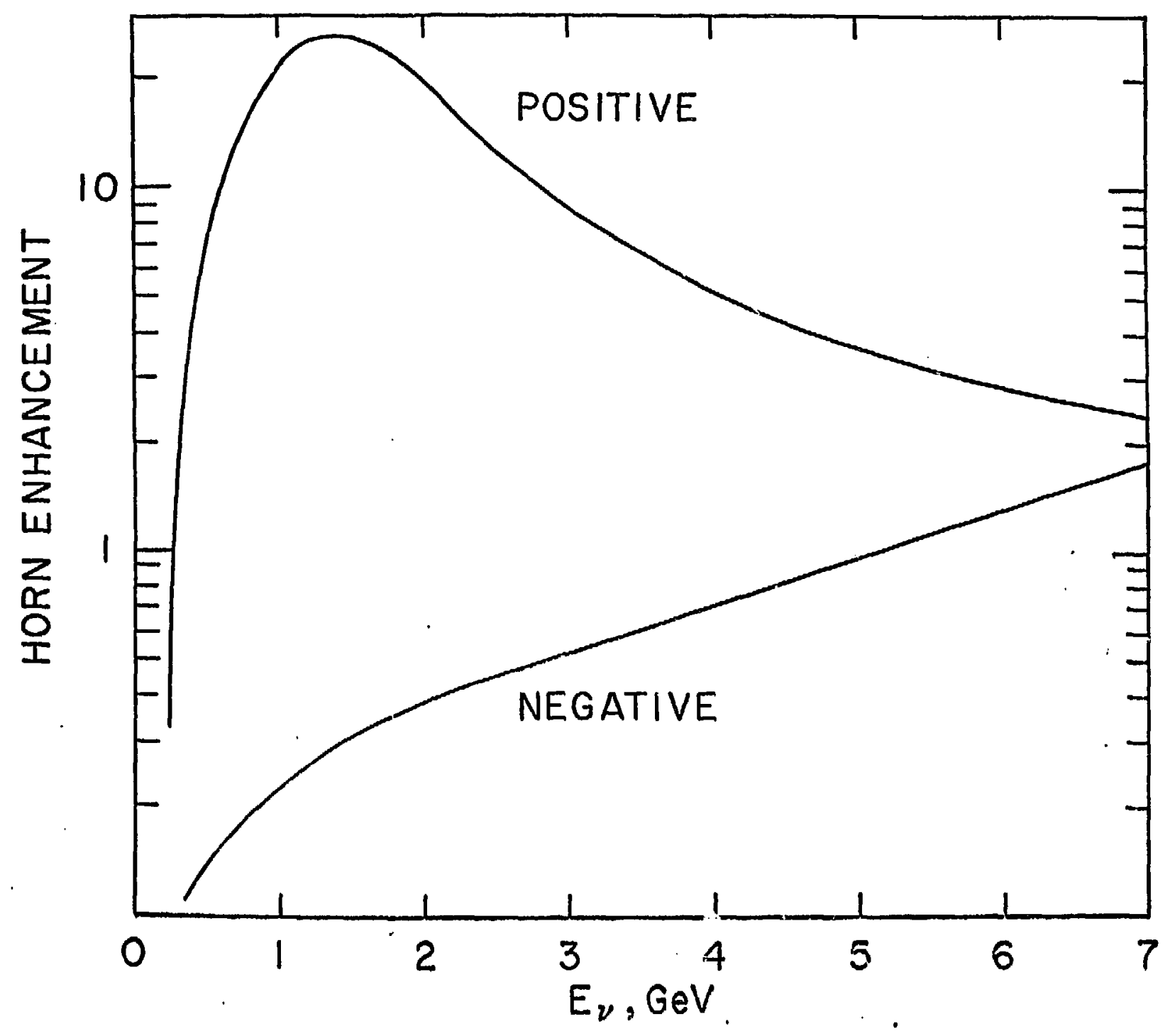




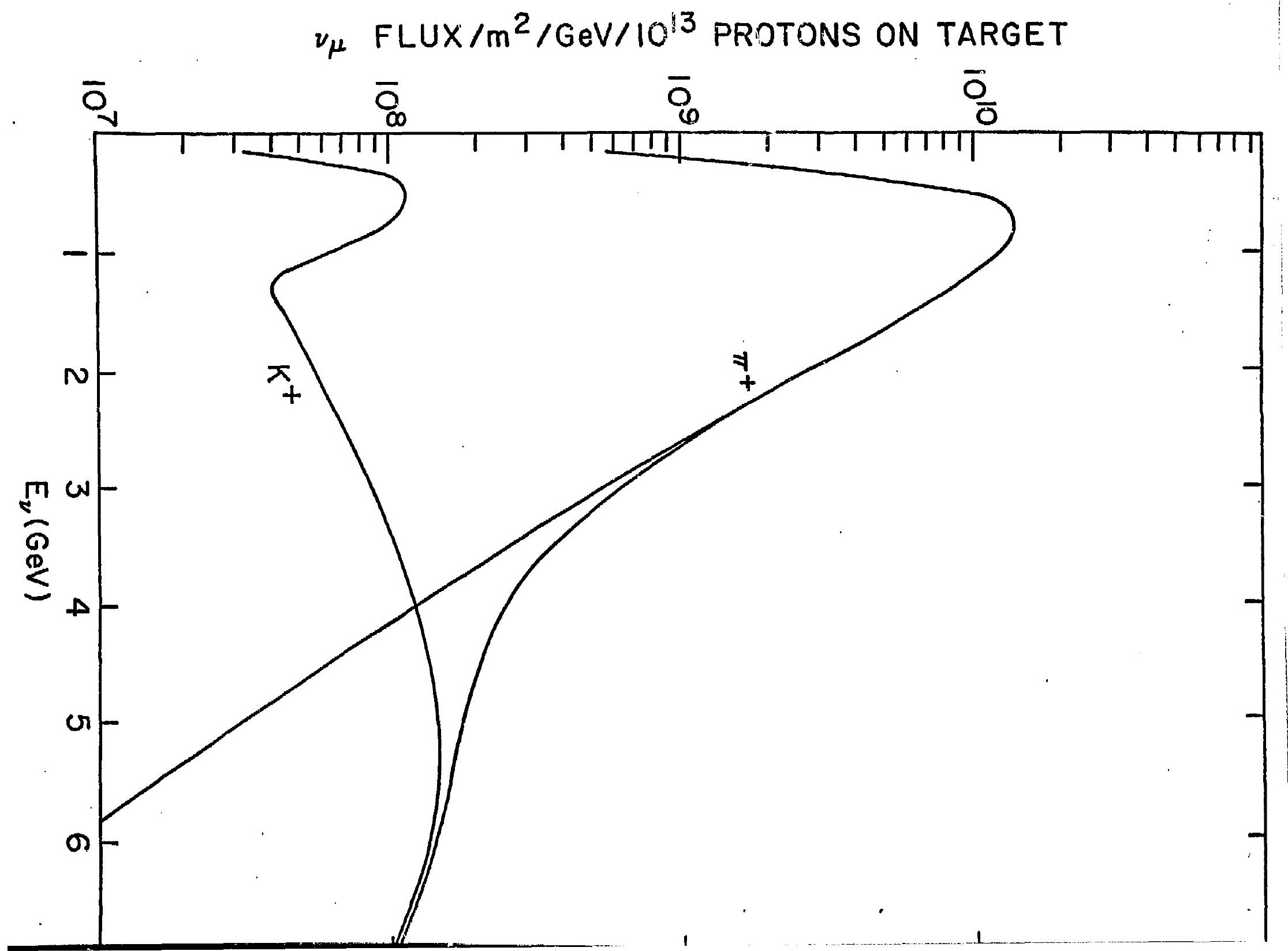




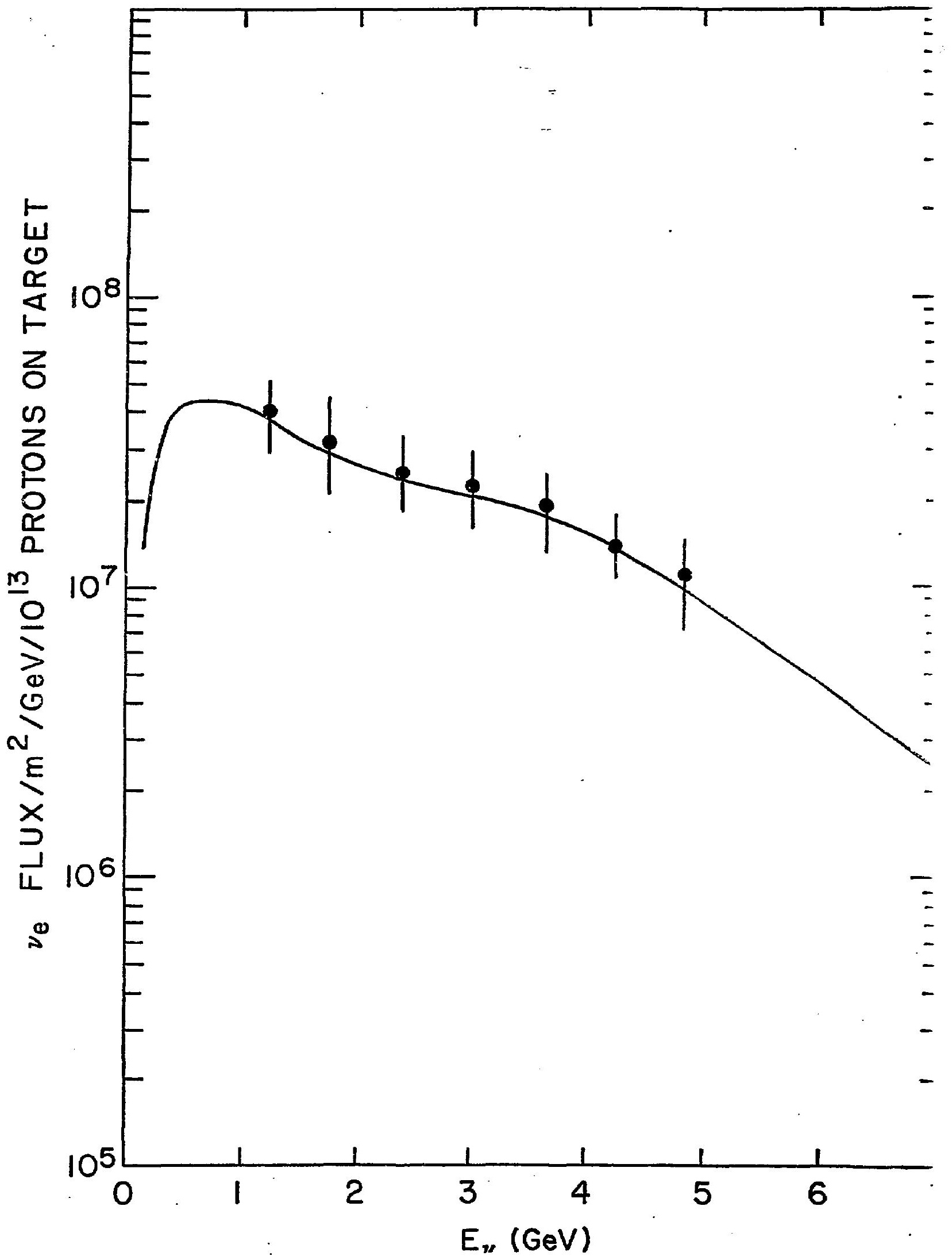

